\title{
NOTE ON LAMBERT'S SERIES
}

\author{
By G. H. HARDY.
}

[Received September 25th, 1913.-Read November 19th, 1913.]

1. In a very interesting memoir "Über Lambertsche Reihen," pub. lished recently in Crelle's Journal," Herr K. Knopp proves the following theorem :-

Suppose that the series

$$
\Sigma \frac{a_{k v+l}}{k \nu+l}(l=0,1,2, \ldots, k-1),
$$

are convergent, so that the series

$$
\sum_{1}^{\infty} a_{n} \frac{x^{n}}{1-x^{n}}
$$

is certainly absolutely convergent for $r=|x|<1$. Let $f(x)$ denote the sum of the latter series for $r<1$, and let

$$
x=r x_{0}=r e^{2 \times \pi / / k},
$$

where $\kappa$ is prime to $k$. Then

$$
\lim _{r \rightarrow 1}(1-r) f(x)=\sum_{1}^{\infty} \frac{a_{k \nu}}{k \nu} .
$$

In its simplest form, when $k=1$, this theorem is the analogue for Lambert's series, that is to say, series of the form

$$
\Sigma a_{n} \frac{x^{n}}{1-x^{n}},
$$

of Abel's theorem on the continuity of power series. I gave a proof of

- Journal filr Math., Vol. 142, p. 283. A less general theorem of the same character was proved by Franel, Math. Annalen, Vol. 52, pp. 543 et seq. 
this special form of the theorem in a paper published some years ago in these Proceedings ;* and in a later papert I stated without proof a generalised form of the result, in which the hypothesis of convergence is replaced by that of summability by some one of Cesàro's means. It is naturally suggested that Knopp's theorem should be capable of a similar generalisation, in which the hypothesis is that of the summability of the various series

$$
\Sigma \frac{a_{k v+l}}{k \nu+l}
$$

That the theorem thus suggested is, in fact, true will appear in the sequel. It is not, however, precisely that which I propose to prove. A little reflection, in fact, shows that Knopp's hypothesis may be replaced by another which is more natural and also slightly more general. His theorem consists in reality of two parts. Let us write

$$
f(x)=\sum_{1}^{\infty} a_{k \nu} \frac{x^{k \nu}}{1-x^{k \nu}}+\sum_{l=1}^{k-1} \sum_{0}^{\infty} a_{k v+l} \frac{x^{k \nu+l}}{1-x^{k \nu+l}}=f_{0}(x)+\sum_{l=1}^{k-1} f_{l}(x),
$$

the first series containing those terms which become infinite as $x \rightarrow x_{0}$. It is clear that the limit assigned by the theorem arises solely from $f_{0}(x)$ : the theorem is, in fact, equivalent to two theorems, expressed respectively by the equations

$$
\begin{aligned}
& \lim (1-r) f_{0}(x)=\Sigma \frac{a_{k v}}{k \nu}, \\
& \lim (1-r) f_{l}(x)=0 .
\end{aligned}
$$

Conditions for the truth of (1) are naturally expressed in terms of the series on the right-hand side; but there is nothing in (2) to suggest the introduction of the $\cdot$ series

$$
\sum \frac{a_{k v+l}}{k v+l} \cdot \ddagger
$$

I propose therefore to modify Knopp's condition. The condition

* Proc. London Math. Soc., Ser. 2, Vol. 4, p. 253.

† Math. Annalen, Vol. 64, p. 91.

† The denominator $k \nu$ arises from the fact that

$$
\lim \frac{1-r}{1-x^{k \nu}}=\lim \frac{1-r}{1-r^{k \nu}}=\frac{1}{k \nu} .
$$

But

$$
\lim \frac{1-v}{1-x^{k \nu+1}}=0 \text {. }
$$

SER. 2. VOL. 13 . No. 1202. 
which I shall suppose satisfied is that

$$
\sum_{\nu=0}^{m} a_{k v+l}=o(m)
$$

This is more appropriate and also more general :* we shall tind, moreover, that the adoption of the more general hypothesis leads to a simplification of the proof.

This modified form of Knopp's theorem is a particular case of Jthe theorem which follows, and the proof of which is the object of this note.

2. Theовem 1.-Let $a_{k v+l}=b_{\nu_{1}}$, and suppose that an integer p exists, such that (i) the series

$$
\Sigma \frac{b_{\nu, 0}}{k \nu}=\Sigma \frac{a_{k \nu}}{k_{\nu}}
$$

is summable $(C, p)$, (ii) the p-th Cesàro sum $B_{v, l}^{n}$, formed from the series $\Sigma b_{v, l}$, satisfies the relation

$$
B_{\nu, l}^{p}=o\left(\nu^{p+1}\right)
$$

Then

$$
\lim _{r \rightarrow 1}(1-r) f(x)=\Sigma \frac{a_{k \nu}}{k \nu} .+
$$

3. This theorem, like Knopp's theorem, is really equivalent to two. We first consider $f_{0}(x)$, and write $y=x^{k}$ (so that $y$ is real), and $c_{\nu}=b_{\nu, 0} / \nu$. Then it is clear that what we have to prove is

Theorear 2.-If $\Sigma c_{\nu}$ is summable $(C, p)$, then

$$
\lim _{y \rightarrow 1} \Sigma c_{\nu} \frac{\nu y^{\nu}(1-y)}{1-y^{\nu}}=\Sigma c_{\nu}
$$

* If $s_{n}$ is the sum of the first $n$ terms of a series $\Sigma u_{n}$ the convergence of $\Sigma\left(u_{n} / n\right)$ involves. $s_{n}=o(n)$, whereas the converse is not true.

† That the series which represents $f(x)$ still converges absolutely for $r<1$ is trivial. That the hypothesis (ii) is more general than the hypothesis that $\Sigma \frac{a_{k_{v}+l}}{k \nu+l}$ is summable $(C, p)$. follows from Theorem 14 of Mr. Littlewood's and my paper " Contributions to the Arithmetio Theory of Series," Proc. London Math. Soc., Ser. 2, Vol. 11, p. 435.

The least values of $p$ for which (i) or (ii) is satisfied may differ according to the value of $l$. If one of them is satisfied for any special $p$, it is satisfed for any greater $p$; there is therefore no objection to supposing all the $p$ 's the same. 
This is the theorem which I stated in my paper in the Math. Annalen already referred to. It may be proved as follows.

Write

$$
y=e^{-u}, \quad \phi_{\nu}(u)=\frac{\nu e^{-\nu u}\left(1-e^{-u}\right)}{1-e^{-\nu u}} .
$$

The result will follow from Theorem 3 of the paper quoted, if we can show that

(1) the differences

$$
\Delta^{\lambda} \phi_{\nu}(u) \quad(0 \leqslant \lambda \leqslant p+1)
$$

can be divided into $q_{\lambda}$ groups of successive terms of the same sign, where $q_{\lambda}$ is a number which may depend upon $u$, but remains less than a constant as $u \rightarrow 0$;

(2) the absolute value of

$$
\left|\nu^{\lambda} \Delta^{\lambda} \phi_{\nu}(u)\right| \quad(0 \leqslant \lambda \leqslant p)
$$

is less than a constant for all values of $\nu$ and $u$ in question.

Let

$$
\Phi(\xi)=\frac{\xi e^{-\xi u}}{1-e^{-\xi u}}=\frac{\xi}{e^{\xi n}-1}=\xi \Psi(\xi) .
$$

Then it is easily verified that

$$
\begin{gathered}
\Psi^{(\lambda)}(\xi)=(-u)^{\lambda}\left\{\frac{A_{\lambda, 1}}{e^{\xi u}-1}+\frac{A_{\lambda, 2}}{\left(e^{\xi u}-1\right)^{2}}+\ldots+\frac{A_{\lambda, \lambda+1}}{\left(e^{\xi u}-1\right)^{\lambda+1}}\right\}, \\
\Phi^{(\lambda)}(\xi)=(-u)^{\lambda-1}\left\{\frac{\lambda A_{\lambda-1,1}-A_{\lambda, 1} \xi u}{e^{\xi u}-1}+\frac{\lambda A_{\lambda-1,2}-A_{\lambda, 2} \xi u}{\left(e^{\xi u}-1\right)^{2}}\right. \\
\left.+\frac{\lambda A_{\lambda-1, \lambda}-A_{\lambda, \lambda} \xi u}{\left(e^{\xi u}-1\right)^{\lambda}}-\frac{A_{\lambda, \lambda+1} \xi u}{\left(e^{\xi u}-1\right)^{\lambda+1}}\right\},
\end{gathered}
$$

where the $A$ 's are positive constants and $A_{\lambda, 1}=1, A_{\lambda, \lambda+1}=\lambda$ ! for all values of $\lambda$. If $\Phi^{(\lambda)}(\xi)=0$, we obtain

$$
(\lambda-\xi u) e^{\lambda \xi u}+\ldots+\ldots=0,
$$

an equation in $\xi u$ whose remaining terms contain powers of $e^{\xi u}$ lower than the $\lambda$-th. It is plain that the number $s$ of positive roots of this equation depends only on $\lambda$. Let us denote these roots by $\eta_{1}, \eta_{2}, \ldots, \eta_{s}$. Then the roots of $\Phi^{(\lambda)}(\xi)=0$ are

$$
\xi=\eta_{1} / u, \quad \eta_{2} / u, \quad \ldots, \quad \eta_{s} / u .
$$

Now

$$
\phi_{\nu}(u)=\left(1-e^{-u}\right) \Phi(\nu),
$$

$$
\Delta^{\lambda} \phi_{\nu}(u)=\left(1-e^{-u}\right) \Delta^{\lambda} \Phi(\nu)=(-1)^{\lambda}\left(1-e^{-u}\right) \Phi^{(\lambda)}(\hat{\xi}),
$$


where $\nu \leqslant \xi \leqslant \nu+\lambda$. Choose $u$ small enough to ensure that no two of $\eta_{1} / u, \eta_{8} / u, \ldots, \eta_{s} / u$ differ by less than $\lambda$, so that the interval $(\nu, \nu+\lambda)$ cen include at most one root of $\Phi^{(\lambda)}(\xi)=0$. As $\nu$ increases from 1 onwards, $\Delta^{\lambda} \phi_{\nu}(u)$ remains of fixed sign until $\nu+\lambda>\xi_{1} / u$. After this alternations may occur, but they must cease as soon as $\nu>\xi_{1} / u$. The total number of possible changes of sign associated with the root $\xi_{1} / u$ is at most $\lambda+1$. Proceeding in this way we see that $\Delta^{\lambda} \phi_{\nu}(u)$ cannot change sign more than $(\lambda+1) s$ times in all. This proves the proposition (1).

In order to prove (2) we must show that

$$
\left|\left(1-e^{-u}\right) \nu^{\lambda} \Delta^{\lambda} \Phi(\nu)\right|
$$

is less than a constant; and this will be so if the same assertion is true of

$$
\left|u \xi^{\lambda} \Phi^{(\lambda)}(\xi)\right| \text {. }
$$

Referring back to the explicit formula for $\Phi^{(\lambda)}(\xi)$, we see that what we have to prove is that the functions

$$
\frac{(\xi u)^{\lambda}}{\left(e^{\xi \mu}-1\right)^{\mu}}(\mu \leqslant \lambda), \quad \frac{(\xi u)^{\lambda+1}}{\left(e^{\xi \mu}-1\right)^{\mu}}(\mu \leqslant \lambda+1)
$$

are less than constants, and this is obvious. Thus (2) is true, and the proof of Theorem 2 is accordingly completed.

4. In order to complete the proof of Theorem 1, we have to show that

$$
f_{l}(x)=\Sigma b_{v, l} \frac{x^{k \nu+l}}{1-x^{k v+l}}=o\left(\frac{1}{1-r}\right) .
$$

The series may be written in the form

$$
\Sigma b_{v, l} \frac{a r^{2 v+l}}{1-\alpha \gamma^{k v+l}},
$$

where $\alpha=e^{2 k \pi r i / k}$. Hence our theorem will follow as a corollary of

TheOREM 3.-If

$$
g(r)=\Sigma c_{\nu} \frac{r^{k \nu+l}}{1-a r^{k \nu+l}},
$$

where $a$ is any number other than a positive number not less than 1 , and the p-th Cesaro sum $C_{\nu}^{p}$ formed from $\Sigma c_{\nu}$ satisfies

therr

$$
C_{\nu}^{p}=o\left(\nu^{p+1}\right) \text {, }
$$$$
g(r)=o\left(\frac{1}{1-r}\right) \text {, }
$$

as $r \rightarrow 1$. 
The proof of this is simple. Let $\rho=r^{k}$ and $\beta=\alpha \imath^{l}$. Then

$$
\begin{aligned}
\Delta \frac{\gamma^{k \nu}}{1-\alpha r^{k \nu+l}} & =\Delta \frac{\rho^{\nu}}{1-\beta \rho^{\nu}}=\frac{(1-\rho) \rho^{\nu}}{\left(1-\beta \rho^{\nu}\right)\left(1-\beta \rho^{\nu+1}\right)}, \\
\Delta^{2} \frac{\gamma^{k \nu}}{1-\alpha r^{k \nu+l}} & =\frac{(1-\rho)^{2} \rho^{\nu}\left(1+\beta \rho^{\nu+1}\right)}{\left(1-\beta \rho^{\nu}\right)\left(1-\beta \rho^{\nu+1}\right)\left(1-\beta \rho^{\nu+2}\right)},
\end{aligned}
$$

and generally

$$
\Delta^{p+1} \frac{r^{k \nu}}{1-\alpha r^{k v+l}}=\frac{(1-\rho)^{p+1} \rho^{\nu} \chi_{p+1}}{\left(1-\beta \rho^{\nu}\right)\left(1-\beta \rho^{\nu+1}\right) \ldots\left(1-\beta \rho^{\nu+p+1}\right)},
$$

where $\chi_{p+1}$ is a polynomial in $\rho$ and $\beta$ whose coefficients depend only on $p$. As $\beta$ satisfies the same condition as $\alpha$, the factors in the :denominator are all greater in absolute value than a constant. Hence

$$
\Delta^{n+1} \frac{\vartheta^{j k \nu}}{1-a \gamma^{k \nu+l}}=(1-\rho)^{p+1} \rho^{\nu} O(1)
$$

and so $\Sigma c_{\nu} \frac{\gamma^{k \nu}}{1-\alpha \gamma^{k \nu+l}}=\Sigma C_{\nu} \Delta \frac{\gamma^{k \nu \nu}}{1-\alpha \gamma^{k \nu+l}}=\ldots=\Sigma C_{\nu}^{p} \Delta^{p+1} \frac{\gamma^{k v_{\nu}}}{1-\alpha \gamma^{\gamma^{k \nu+l}}}$

$$
=O(1-r)^{p+1} \Sigma o\left(\nu^{p+1}\right) r^{k \nu}=o\left(\frac{1}{1-r}\right) \text {. }
$$

5. It is easy to verify that all our conditions are satisfied if

$$
a_{n}=(-1)^{n} n^{s}
$$

where $s$ is any number real or complex, and $k$ is odd. Hence, when $x$ approaches the point $e^{2 \kappa \pi i / k}$ along a radius vector,

$$
f(x)=\Sigma \cdot \frac{(-1)^{n} n^{s} x^{n}}{1-x^{n}} \sim \frac{k^{s-1}}{1-r} \Sigma(-1)^{k \nu} \nu^{s-1} .
$$

If $k$ is even, the series on the right is no longer summable; and $f(x)$ is, in fact, of higher order than $1 /(1-r)$. Suppose, e.g., that $s$ is positive, and $k=2, \kappa=1$, so that $x \rightarrow-1$. Then

$$
\begin{gathered}
f(x)=\Sigma \frac{n^{s} \cdot y^{n}}{1-y^{n}} \sim \frac{\Gamma(s+1) \xi(s+1)}{(1-y)^{s+1}}, \\
y=-x \rightarrow 1 .^{*}
\end{gathered}
$$

6. It is natural to suppose that Theorems 1-3 retain their validity 
when $x \rightarrow x_{0}$ along any "Stolz-path," i.e., any curve which has a continuous tangent and does not touch the unit circle. Knopp* has extended his theorem to this case, but only under a narrower hypothesis, viz., that the series $\Sigma\left|\frac{a_{n}}{n}\right|$ is convergent. It is quite easy to see that Theorem 3 is still true under the more general hypothesis; but to make the corresponding extension of Theorem 2 (and so of Theorem 1) appears to be a less simple matter. The proof would presumably be based upon a theorem of Dr. Bromwich $†$ which includes as a special case the theorem of $\min \theta$ used in $\S 3$.

* L.c., $\$ 1$, p. 800.

$\dagger$ Math. Annalen, Vol. 65, p. 859. 